\title{
Mass-specific optical absorption coefficients and imaginary part of the complex refractive indices of mineral dust components measured by a multi-wavelength photoacoustic spectrometer
}

\author{
N. Utry ${ }^{1}$, T. Ajtai ${ }^{2}$, M. Pintér ${ }^{1}$, E. Tombácz ${ }^{3}$, E. Illés ${ }^{3}$, Z. Bozóki ${ }^{2}$, and G. Szabó ${ }^{2}$ \\ ${ }^{1}$ Department of Optics and Quantum Electronics, University of Szeged, Szeged, Hungary \\ ${ }^{2}$ MTA-SZTE Research Group on Photoacoustic Spectroscopy, Szeged, Hungary \\ ${ }^{3}$ Department of Physical Chemistry and Materials Science, University of Szeged, Szeged, Hungary
}

Correspondence to: T. Ajtai (ajtai@titan.physx.u-szeged.hu)

Received: 25 June 2014 - Published in Atmos. Meas. Tech. Discuss.: 8 September 2014

Revised: 8 December 2014 - Accepted: 17 December 2014 - Published: 22 January 2015

\begin{abstract}
Mass-specific optical absorption coefficients (MACs) and the imaginary part $(\kappa)$ of the refractive indices of various mineral dust components including silicate clays (illite, kaolin and bentonite), oxides (quartz, hematite and rutile), and carbonate (limestone) were determined at the wavelengths of 1064, 532, 355 and $266 \mathrm{~nm}$. The MAC values were calculated from aerosol optical absorption coefficients measured by a multi-wavelength photoacoustic (PA) instrument, the mass concentration and the number size distribution of the generated aerosol samples as well as the size transfer functions of the measuring instruments. Values of $\kappa$ were calculated from the measured and particle-loss-corrected data by using a Mie-theory-based retrieval algorithm. The determined values could be used for comparisons with calculated wavelength-dependent $\kappa$ values typically deduced from bulkphase measurements by using indirect measurement methods. Accordingly, the presented comparison of the measured and calculated aerosol optical absorption spectra revealed the strong need for standardized sample preparation and measurement methodology in case of bulk-phase measurements.
\end{abstract}

\section{Introduction}

Mineral dust (MD) in the atmosphere affects the radiative budget of the Earth both directly and indirectly. It absorbs and scatters the solar and terrestrial radiations in the nearinfrared-visible-near-UV wavelength range, and its overall forcing effect is influenced by its chemical composition, tem- poral and spatial distribution as well as its optical properties. As a consequence of this complex dependence, current knowledge on both the sign and the magnitude of the MDinduced radiative forcing is largely uncertain (Claquin et al., 1998). Some studies estimate that the forcing effect of MD compares to or even exceeds the cumulative forcing effect of all other aerosol originating from anthropogenic sources (Sokolik and Toon, 1996). Therefore, there is a strong need for measurement methods which can characterize the optical properties of MD accurately. This is especially important in case of its anthropogenic fraction, which is estimated to make up about $30-50 \%$ of the total atmospheric MD load (Tegen and Fung, 1995).

The wavelength-dependent optical properties of MD are represented by the complex refractive index $(R i=n+i \kappa)$, where $n$ and $\kappa$ are directly associated with scattering and absorption, respectively. Unfortunately, $R i$ and especially its imaginary part $(\kappa)$ is notoriously difficult to be measured because unlike $n$, which is generally well constrained and only slightly (if at all) varies with wavelength, and is almost completely composition independent, $\kappa$ has a strong chemical composition dependence, and might vary over 3 orders of magnitude in the whole climate-relevant spectral region (McConnell et al., 2010; Otto et al., 2007).

Generally speaking, light absorption is one of the most difficult aerosol parameters to be measured even when optical absorption has the same magnitude as scattering (i.e. the single-scattering albedo (SSA) is around 0.5). This is typically the case for black carbon aerosol with $\mathrm{SSA} \approx 0.6$. For 
this type of strongly absorbing aerosol the widely adopted methods for aerosol absorption measurement - i.e. either the differential method based on the simultaneous measurement of scattering and extinction, or methods based on transmission measurement on filter accumulated aerosol (Moosmüller et al., 2009) - work quite well at least in case of large aerosol concentrations. However, the former method has limited sensitivity, while the latter one has large uncertainties due to the spurious effect of filter-light interaction. Compared to this already challenging measurement task, absorption measurements on MD samples are even much more difficult due to the fact that $\kappa$ is typically orders of magnitude smaller than $n$ (i.e. the SSA is typically higher than 0.95). In this case the above-mentioned methods are no longer reliable.

Accurate optical absorption data are needed not only for modelling the effect of MD in the atmosphere by calculating radiative transfer properties such as optical thickness, singlescattering albedo, or asymmetry parameter but also for studying the variation of these parameters due to the variations of MD composition. The geographical location of the emission site was found to strongly influence the composition of the emitted MD (Claquin et al., 1998; Nickovic et al., 2012). Various efforts have already been made to calculate the optical properties of MD from the optical properties of its components (e.g. by using the effective medium approximation (Sokolik et al., 1993; Sokolik and Toon, 1999)). Most often these calculations are based on the Mie theory, and approximate the particles as being spherical and homogeneous. In these calculations the size distribution and the $R i$ of each component play a crucial role. Although refractive indexes of most abundant MD components are measured and tabulated over broad, climate-relevant wavelength ranges, these data are typically derived from bulk-phase indirect measurements, wherefore they have limited reliability. Furthermore, these measurement results are not validated by aerosol-phase measurements (i.e. the actual microphysical aerosol conditions such as size and morphology are neither taken into account nor validated). Very often $R i$ data (and especially $\kappa$ ) differ significantly even when measured at the same or very close wavelengths in the bulk phase due to the differences in the applied measurement methodology and sample treatment (Rao, 2002; Arakawa et al., 1997). There are serious concerns especially about the reliability of $\kappa$ values determined by the most commonly applied diffuse reflectancebased techniques. Claquin et al. (1998) found that the variation of $\kappa$ determined by different methodologies manifests in significant uncertainties in radiative forcing calculations, and thus not only the magnitude but also the sign of the calculated radiative forcing parameter is uncertain.

Another potential application of accurate optical absorption data in a wide wavelength range could be the so-called inverse problem (i.e. the determination of the chemical composition and thus the sources of MD (Müller et al., 2011) from its measured optical properties). This analysis is based on the fact that unlike scattering, which is mainly driven by aerosol size and morphology, absorption has rather strong sensitivity to the chemical composition too (Andreae and Gelencsér, 2006; Moosmüller et al., 2009).

The photoacoustic (PA) measurement technique has already proved its applicability under laboratory and field conditions in case of strongly absorbing aerosol components such as black carbon, brown carbon and HULIS (humiclike substances) (Andreae and Gelencsér, 2008; Cappa et al., 2008; Lack et al., 2012; Chakrabarty et al., 2010; Moosmüller et al., 2012; Ajtai et al., 2010; Utry et al., 2014). The accuracy of the method stems from both its scattering insensitivity and its filter-free sampling (measurement made on the aerosol on its natural, suspended state) (Andreae, 2001). On the other hand, application of the PA technique for the measurement of MD or MD components is a real challenge not only because of the high SSA and strong wavelength dependence of $\kappa$ as described above, but also because of the relatively large size of the measured particles. Indeed, while the typical size of atmospheric black and brown carbon is in the range of a few hundred nanometres or even below, the size of the MD particles is mostly in the micron range. Consequently, the size transfer function of the detection system has to be taken into account accurately, especially for large particles, since already a few of them can considerably modify the aerosol mass concentration in the PA cell with which the PA signal is linearly proportional as described below.

Photoacoustic determination of optical absorption by aerosol is based on the absorption of periodically modulated light and the subsequent non-radiative relaxation of excited aerosol particles. Due to the high thermal conductivity and the small size of climate-relevant ambient aerosol particles, the energy absorbed by them is transferred promptly (adiabatically) to the surroundings. The subsequent thermal expansion induces periodic pressure variation, which is proportional to the concentration and the absorption cross section of light-absorbing particles, can be detected and converted into a measurable electronic signal by an acoustic transducer (typically a microphone). The optical absorption coeffitient (OAC) can be deduced from the measured PA signal by using a conversion factor determined by an independent calibration of the PA system. The major advantages of this method compared to the alternatives are selectivity, sensitivity, and accuracy. Selectivity means that PA is not only a zero background technique (i.e. in the absence of light absorption no PA signal is generated) but in addition to that the PA response is inherently unaffected by the non-absorbing aerosol particles present in the sampled aerosol stream, and also by the non-absorbing compounds of the individual aerosol particles (Moosmüller et al., 2009; Bozóki et al., 2011). The accuracy of the recently published PA instruments for aerosol measurement is in the range of a few percent, which is at least an order of magnitude better than that of the most commonly used filter-based instruments, even when their readings are 
corrected posteriorly after the measurement (Andreae and Gelencsér, 2006).

To the best of our knowledge, this paper presents the aerosol-phase mass-specific optical absorption coefficient (MAC) spectra of the most abundant mineralogical MD components for the first time. However it is worth noting that single-scattering albedo of several mineral dust samples was also previously determined by the combination of multiwavelength photoacoustic spectroscopy and nephelometry (Moosmüller et al., 2012). These spectra are measured by our recently developed multi-wavelength photoacoustic spec-

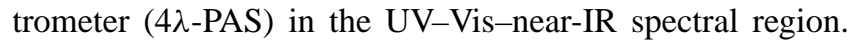
Furthermore, the Mie-theory-based simulated OAC spectra are generated by using bulk-phase $R i$ values derived from published literature and the measured number size distributions corrected by the size transfer function of the measuring system. These theoretical curves are compared with measured OAC spectra, and based on these comparisons the PA method is arguably a powerful tool for verifying theoretical calculations.

\section{Experimental and methods}

\subsection{Sample preparation}

In this study MD components, phyllosilicates (illite, kaolin and bentonite), oxides (quartz, hematite and rutile), and carbonate (limestone) were investigated. High-purity illite was obtained from a Hungarian source Füzérradvány and measured as received. Its mineral composition is illite 75 , montmorillonite 5, quartz 14, kaolinite 1 and feldspar $4 \%$ and its chemical composition is $\mathrm{SiO}_{2} 47.5, \mathrm{Al}_{2} \mathrm{O}_{3} 29.0, \mathrm{Fe}_{2} \mathrm{O}_{3}$ 1.2, $\mathrm{CaO} 1.8, \mathrm{MgO} 3.0, \mathrm{~K}_{2} \mathrm{O} 6.8, \mathrm{Na}_{2} \mathrm{O}$ 1.1, loss of ignition 8.5 and total $98.9 \%$. Kaolin was obtained from Zettlitz (Germany). It contains clay mineral kaolinite as the main component up to $90 \%$, accompanied by some micaceous minerals and small amounts of quartz. Chemical composition of kaolin is $\mathrm{MgO} 0.27, \mathrm{CaO} 0.29, \mathrm{SiO}_{2} 46.9, \mathrm{Al}_{2} \mathrm{O}_{3}$ 37.4, $\mathrm{Fe}_{2} \mathrm{O}_{3}$ 0.65, $\mathrm{K}_{2} \mathrm{O}$ 0.84, $\mathrm{Na}_{2} \mathrm{O} 0.44, \mathrm{TiO}_{2} 0.18, \mathrm{H}_{2} \mathrm{O} 12.95$ and total $99.92 \%$. We used a fine-grained washed kaolin sample with a characteristic particle size of 1.2-1.4 $\mu \mathrm{m}$. The source of montmorillonite was bentonite from Mád (Hungary). Its mineral composition is montmorillonite 86 , quartz 3 , feldspar $5 \%$ and its chemical composition is $\mathrm{SiO}_{2}$ 63.8, $\mathrm{A}_{2} \mathrm{O}_{3}$ 16.0, $\mathrm{Fe}_{2} \mathrm{O}_{3}$ 5.0, $\mathrm{CaO} 2.4, \mathrm{MgO} 1.2, \mathrm{~K}_{2} \mathrm{O} 0.8, \mathrm{Na}_{2} \mathrm{O}$ $1.1, \mathrm{TiO}_{2} 0.6$, loss of ignition 8.9 and total $99.8 \%$. The mined bentonite had a grain size of less than $90 \mu \mathrm{m}$, activated by $5 \% \mathrm{Na}_{2} \mathrm{CO}_{3}$ and enriched in montmorillonite. A high-purity quartz sample $\left(\mathrm{SiO}_{2}>99 \%\right)$ was ground and the sieve fraction $<200 \mu \mathrm{m}$ was used. Hematite $\left(\mathrm{Fe}_{2} \mathrm{O}_{3}\right)$ produced by Reanal Private Ltd. (Hungary) had a degree of purity $>95 \%$. Rutile (Bayertitan R-U-2) produced by Bayer AG contained $98 \% \mathrm{TiO}_{2}$; it is an alumina lattice modified sample, its relative light-scattering power is 96 and its specific gravity is

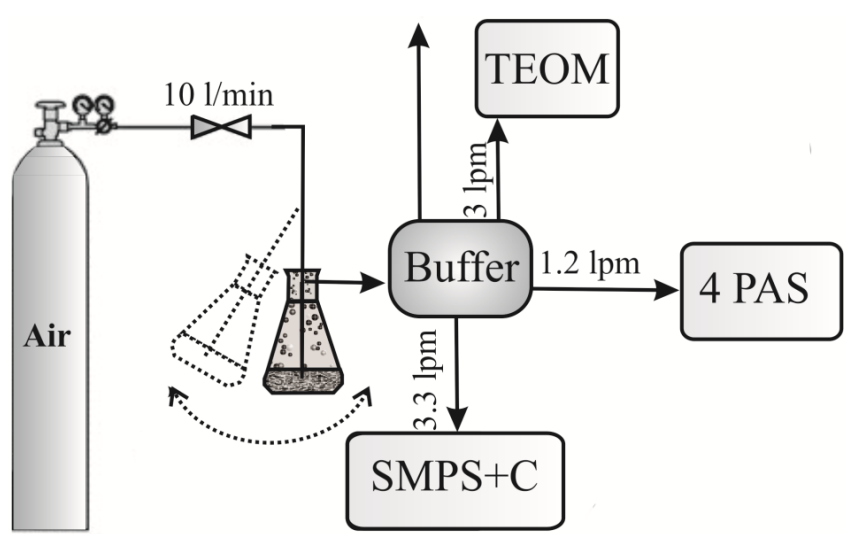

Figure 1. Schematic diagram of the experimental set-up (the vessel with dotted contour line and the arrow indicates that it is being shaken).

$4.1 \mathrm{~g} \mathrm{~cm}^{-3}$. Limestone (calcium carbonate with $\mathrm{CaCO}_{3}$ content $98 \%$ ) was collected in the Pilis Mountains (Hungary), and then ground to produce fine powder.

\subsection{Sample generation}

MD particles were re-dispersed from the prepared powder sample and added to the buffer chamber for instrument sampling in the experimental set-up depicted in Fig. 1.

Prior to the measurements, aerosol samples were desiccated in a vessel partly filled with silica gel for at least $24 \mathrm{~h}$. A small amount of mineral powder was placed into the Erlenmeyer vessel and then into an automatic shaker. Synthetic air was blown through the shaker at a constant flow rate of $10 \mathrm{Lpm}$. The re-dispersed aerosol was fed into a buffer chamber. Pressure was kept at atmospheric level by an exhaust pipe implemented into the buffer chamber. Each instrument sucked the aerosol loaded air mass directly from the chamber. The relative humidity $(\mathrm{RH})$ of the air stream including suspended particles in the buffer chamber was measured during the whole measurement campaign and found to be below $\mathrm{RH} \sim 30$ in each sample. All the presented measurement results were performed at room temperature and atmospheric pressure.

\subsection{Instrumentation for aerosol characterization}

The OAC was measured simultaneously at four wavelengths by a multi-wavelength photoacoustic spectrometer ( $4 \lambda$-PAS). It contained four measuring cells with each of them being illuminated through one of the operational wavelengths of 266 , 355,532 or $1064 \mathrm{~nm}$. With the help of a magnetic valve controlled by the electronics of the system, the sample flow path through the analyser was regularly switched in a way that the purging gas with the aerosols would flow through a filter before entering the PA cells. In this way the background PA signal (i.e. the signal generated with zero aerosol concentration) 
was measured and subtracted from the PA signal generated by the aerosol. This repeated background subtraction was necessary because laser beams can have some pointing instability, and thus the background PA signal may vary in time. Furthermore, besides aerosol and background measurements the analysers also have a calibration mode during which the optical set-up is modified with the help of flip-up mirrors so that each cell is illuminated with the $532 \mathrm{~nm}$ beam and the PA signal is measured at different $\mathrm{NO}_{2}$ concentrations. The sensitivity of each cell was calculated from the slope of the line which was fitted to the $\mathrm{NO}_{2}$ concentrations vs. the PA signal points divided by the light power going through the cell in the calibration mode, and also by the optical absorption coefficient, which corresponded to the unit concentration of $\mathrm{NO}_{2}$ at the measurement wavelength. Multiplying the sensitivity of a given cell with the light power going through the cell in the measurement mode gives the conversion factor with which the background-subtracted aerosol-generated PA signal has to be multiplied in order to calculate the OAC from the PA measurement. This calibration protocol ensures the wavelength- and composition-independent determination of the OAC. The accuracy of the instrument is between 4 and $10 \%$ depending on the applied wavelength, where the decreased accuracy towards the shorter wavelengths is due to the decreased accuracy of laser power measurement. In the measurement mode a complete 15 min long measurement cycle includes a 10 min sampling and a 5 min background measurement period. Generally, eight measurement cycles were used for data evaluation. The initial transient period was not evaluated. Depending on the investigated mineral dust component and the applied wavelength, the fluctuation of the PA signal including concentration instability was found to be in between 15 and $22 \%$. The number concentration and size distribution of the dust minerals were measured simultaneously in the size ranges of 5 to $950 \mathrm{~nm}$ and $350 \mathrm{~nm}$ to $32 \mu \mathrm{m}$ with a scanning mobility particle sizer (SMPS, GRIMM system Aerosol Technik, Germany, type SMPS) and an optical particle counter (OPC), respectively. The SMPS included a condensation nucleus counter (CPC Model \#5.400) and a classifier "Vienna"-type long differential mobility analyzer (LDMA, Model \#5.500). The LDMA separates particles according to their mobility by balancing their drag and electrical force on the equally charged aerosol stream. The sized particles are then sent to CPC for size-segregated number density measurement. The multiple charge correction defined by the ISO 15900 recommendation was used in the data evaluation during the whole measurement campaign to further increase the reliability of the measured data. Coinciding correction of the measured data was made in order to minimize the shielding effect that occurs when two or more particles arrive in the detection chamber simultaneously. The sheath and aerosol flow rate were set to 3.0 and $0.3 \mathrm{Lpm}$, respectively. In the OPC the sample air with various size particles was drawn constantly through the flat light beam produced by a focused laser diode. Each signal was then counted and classified in different size channels by an integrated pulse height analyser. The differences between the data measured by SMPS and OPC in the overlapping size domain were found to be inside the cumulative uncertainty range of the instrumentations (i.e. below $10 \%$ ); therefore, it was neglected during the data evaluation. Aerosol mass concentration was measured by a tapered element oscillating microbalance (TEOM, Rupprecht and Patashnick, Model 1400a). TEOM incorporates an inertial balance that directly measures the mass collected on an exchangeable filter cartridge by monitoring the corresponding frequency changes of a tapered element (Patashnick et al., 2002). The sample flow rate and the average time were set to be $3 \mathrm{Lpm}$ and $10 \mathrm{~min}$, respectively.

In addition to the online measurements, the dispersed MD components were collected on quartz filters for morphology analysis by scanning electron microscopy (SEM).

\subsection{Calculations}

In order to determine the number size distributions of aerosol samples within the central acoustic resonator of the PA cells where the PA signal generation occurs, as well as within each instrument connected to the aerosol generation buffer volume, the measured SMPS data were corrected with the calculated size transfer function of the experimental set-up by using the particle loss calculator (http://www.mpch-mainz. mpg.de/ drewnick/PLC; Von der Weiden et al., 2009). The model behind this code takes into account most of the physical phenomena which can cause particle losses, such as diffusion, sedimentation, turbulent deposition as a function of dimensions, flow rate, angle of bending, etc. The calculated high end $(50 \%)$ cut-off diameter in the size transfer function of the PA system is at $1.2 \mu \mathrm{m}$ (Utry et al., 2014). The uncertainty of the particle loss calculator was determined independently in another study (von der Weiden et al., 2009). Since the experimental set-up presented here is much less complicated (i.e. devoid of double bend configuration with sharp curvature) than the one used in the validation procedure and all the applied instruments use low flow sampling in the laminar flow region where the reliability of this packet loss concealment algorithm is more adequate, we used this uncertainty in this study.

For calculating the mass-specific optical absorption coefficient (MAC) for each MD component the following quantities were used: the photoacoustically measured OAC; the mass concentration of the aerosol measured by the TEOM; and the mass concentrations calculated for the measured size distribution ratio multiplied with the aerosol mass ratio calculated from the measured size distribution with and without taking into account the particle losses. Based on these quantities MAC was calculated as follows: 
$\mathrm{MAC}=\frac{\mathrm{AOC}\left[\int_{0}^{\infty} D_{\mathrm{p}}^{a} n_{N} \mathrm{~d} D_{\mathrm{p}}\right]_{\text {cor }}}{m_{\mathrm{TEOM}}\left[\int_{0}^{\infty} D_{\mathrm{p}}^{a} n_{N} \mathrm{~d} D_{\mathrm{p}}\right]_{\text {meas }}}$,

where $m_{\text {TEOM }}$ is the mass concentration measured by TEOM, while the $\left[\int_{0}^{\infty} D_{\mathrm{p}}^{a} n_{N} \mathrm{~d} D_{\mathrm{p}}\right]_{\text {meas }}$ and the $\left[\int_{0}^{\infty} D_{\mathrm{p}}^{a} n_{N} \mathrm{~d} D_{\mathrm{p}}\right]_{\text {cor }}$ are the measured and the particleloss-corrected volume concentrations, respectively.

For each MD component an optical absorption spectrum was calculated by using the Mie theory. This calculation also takes into account the measured and particle-loss-corrected size distribution. Furthermore, $R i$ values, which are most often applied (e.g. in effective medium approximation-based calculations), were obtained from various publications as follows:

- Bentonite: in this study we used the optical constants of montmorillonite, which is the main constituent of bentonite. Data of Arakawa et al. (1997) derived from transmission measurement on a thin film were used.

- Kaolin: both the data of Egan and Hilgeman (1979) and Arakawa et al. (1997) made on thin films by diffuse scattering and transmission measurement, respectively, were used.

- Illite: we used data from Egan and Hilgeman (1979). They applied two measurement methods: reflectance and diffuse transmittance measurements performed on a pressed pellet and on a thin section, respectively.

- Rutile: two Ri data sets were obtained from the SOPRA N\&K database (http://www.sopra-sa.com/more/ database.asp). Both of them were deduced from ellipsometric measurements conducted on thin films.

- Hematite: data from Querry (1985) which were based on diffuse reflectance from a bulk material were used. Furthermore, based on the segregated spectra for $\mathrm{o}$ - and e-rays, the sample anisotropy was taken into account by using the spectral averaging method. First the individual spectra for both o-ray and e-ray indices were calculated; then - since there are two equivalent axes for o-ray and only one for e-ray - a weighted average of these individual spectra in the form of [(2/3) o-ray + (1/3) e-ray] was calculated.

- As far as quartz and limestone are concerned, unfortunately no $\kappa$ data were found in the literature for the studied wavelength range.

Values of $\kappa$ were also calculated from the measured and particle-loss-corrected data by using a simple Mie-theorybased retrieval algorithm (Guyon et al., 2003; Hoffer et al.,
2006). First, the Mie code takes the $n$ values from the literature and the measured and particle-loss-corrected size distribution data as input parameters, and then calculates the absorption coefficient. Thereafter this value is compared to the measured absorption coefficients and following that the initial $\kappa$ value is increased stepwise until the calculated and measured optical coefficients agree to within $0.5 \%$. The $\kappa$ value associated with this agreement represents the imaginary part of the investigated sample. However, it is worth noting that although the application of Mie theory for nonspherical particles limits the reliability of the computed data, many prior works used this approach to calculate the spectral responses of dust minerals (Conant et al., 2003; DeSouzaMachado et al., 2006; Moffet and Prather, 2005; Wang et al., 2002). This is because the most widely used shape-sensitive models such as T-matrix or DDA (discrete dipole approximation) are also not using the real morphology of the investigated aerosol sample, but they are based on mathematically well characterized geometrical approximation (Kalashnikova and Sokolik, 2004; Tegen and Lacis, 1996). Kalashnikova and Sokolik demonstrated that the deviation between the spherical and non-spherical approaches becomes significant above the approximate size parameter of 5 and only in case of the asymmetry parameter and the scattering phase function, which are mainly governed by the coherent scattering physical process. However, for the absorption, which is an incoherent physical phenomenon, the deviation is much less significant even above size parameter higher than about 5 .

\section{Results and discussion}

The MACs and the imaginary parts of the complex refractive indices deduced from the measured data are listed in Tables 1 and 2, respectively. Number size distributions can be seen in Fig. 2. Thick lines represent those measured by the OPC and SMPS, while thin lines are derived from the same OPC+SMPS measurements but corrected with the calculated size transfer function of the PA system (i.e. these are the number size distributions that are assumed to be within the PA cells). Figure 3 shows the photoacoustically measured OACs and the corresponding calculated optical absorption spectra. In some cases the results of the calculations are also presented without taking into account the particle losses in the PA system for comparison purposes. SEM pictures of the measured particles are shown in Fig. 4.

The SEM pictures are largely similar to those reported in the literature (Bohor and Hughes, 1971; Dong et al., 2014) and show that apart from rutile, which has a more or less compact and spheroid shape, all other particles show a high degree of irregularity with versatile and unclassifiable shapes.

Although all the instruments were placed as close to the buffer chamber as possible to minimize the curves and 
Table 1. Mass-specific optical absorption coefficients of the measured MD components.

\begin{tabular}{|c|c|c|c|c|}
\hline \multirow[b]{2}{*}{ Mineral component } & \multicolumn{4}{|c|}{ Mass-specific optical absorption coefficients $\left[\mathrm{m}^{2} \mathrm{~g}^{-1}\right]$} \\
\hline & $266 \mathrm{~nm}$ & $355 \mathrm{~nm}$ & $532 \mathrm{~nm}$ & $1064 \mathrm{~nm}$ \\
\hline Illite & $1.0 \times 10^{-1} \pm 2 \times 10^{-2}$ & $1.9 \times 10^{-2} \pm 2 \times 10^{-3}$ & $5.1 \times 10^{-3} \pm 5 \times 10^{-4}$ & $2.5 \times 10^{-3} \pm 5 \times 10^{-4}$ \\
\hline Kaolin & $4.8 \times 10^{-1} \pm 7 \times 10^{-2}$ & $2.3 \times 10^{-1} \pm 3 \times 10^{-2}$ & $2.1 \times 10^{-2} \pm 3 \times 10^{-3}$ & $8.8 \times 10^{-3} \pm 1 \times 10^{-3}$ \\
\hline Bentonite & $3.1 \times 10^{-1} \pm 6 \times 10^{-2}$ & $5.8 \times 10^{-2} \pm 1 \times 10^{-2}$ & $5.2 \times 10^{-3} \pm 1 \times 10^{-3}$ & $4.6 \times 10^{-3} \pm 9 \times 10^{-4}$ \\
\hline Hematite & $5.3 \times 10^{-1} \pm 1 \times 10^{-1}$ & $5.7 \times 10^{-1} \pm 1 \times 10^{-1}$ & $5.4 \times 10^{-1} \pm 1 \times 10^{-1}$ & $3.4 \times 10^{-2} \pm 8 \times 10^{-3}$ \\
\hline Rutile & $3.5 \times 10^{-1} \pm 5 \times 10^{-2}$ & $7.1 \times 10^{-1} \pm 1 \times 10^{-1}$ & $2.2 \times 10^{-3} \pm 3 \times 10^{-4}$ & $1.4 \times 10^{-3} \pm 2 \times 10^{-4}$ \\
\hline Quartz & $1.0 \times 10^{-2} \pm 2 \times 10^{-3}$ & $5.8 \times 10^{-3} \pm 1 \times 10^{-3}$ & $2.3 \times 10^{-3} \pm 4 \times 10^{-4}$ & $2.0 \times 10^{-3} \pm 4 \times 10^{-4}$ \\
\hline Limestone & $5.3 \times 10^{-3} \pm 5 \times 10^{-4}$ & $6 \times 10^{-3} \pm 7 \times 10^{-4}$ & $5.5 \times 10^{-4} \pm 1 \times 10^{-4}$ & $3.8 \times 10^{-4} \pm 4 \times 10^{-5}$ \\
\hline
\end{tabular}

Table 2. Imaginary parts of the complex refractive indices $(\kappa)$ of the measured MD components. For the sake of clarity these data are supplemented with the $n$ and $\kappa$ values taken from the literature, where the former ones are used in the $\kappa$ calculation while the latter ones are shown in this table for inter-comparison purposes.

\begin{tabular}{|c|c|c|c|c|c|c|c|c|c|}
\hline \multirow[t]{2}{*}{ Mineral component } & & $\lambda_{1}$ & $266 \mathrm{~nm}$ & $\lambda_{2}$ & $355 \mathrm{~nm}$ & $\lambda_{3}$ & $532 \mathrm{~nm}$ & $\lambda_{4}$ & $1064 \mathrm{~nm}$ \\
\hline & & $n$ & $k$ & $n$ & $k$ & $n$ & $k$ & $n$ & $k$ \\
\hline \multirow{2}{*}{ Illite } & reference $^{1}$ & 1.42 & $2.0 \times 10^{-3}$ & 1.41 & $1.5 \times 10^{-3}$ & 1.41 & $8.3 \times 10^{-4}$ & 1.39 & $1.2 \times 10^{-3}$ \\
\hline & calculated & & $1.1 \times 10^{-2}$ & & $1.7 \times 10^{-3}$ & & $7.0 \times 10^{-4}$ & & $8 \times 10^{-4}$ \\
\hline \multirow[t]{2}{*}{ Kaolin } & reference $^{2}$ & 1.67 & $4.0 \times 10^{-2}$ & 1.61 & $9.1 \times 10^{-3}$ & 1.57 & $6.3 \times 10^{-3}$ & 1.56 & $5.9 \times 10^{-3}$ \\
\hline & calculated & & $4.2 \times 10^{-2}$ & & $6.2 \times 10^{-3}$ & & $7.0 \times 10^{-4}$ & & $9.0 \times 10^{-4}$ \\
\hline \multirow[t]{2}{*}{ Bentonite } & reference $^{2}$ & 1.64 & $3.2 \times 10^{-2}$ & 1.58 & $2.4 \times 10^{-2}$ & 1.54 & $2.4 \times 10^{-3}$ & 1.52 & $1.1 \times 10^{-3}$ \\
\hline & calculated & & $3.1 \times 10^{-2}$ & & $2.5 \times 10^{-2}$ & & $2.0 \times 10^{-3}$ & & $1.3 \times 10^{-3}$ \\
\hline \multirow[t]{2}{*}{ Hematite } & reference $^{3}$ & 1.94 & $1.25 \times 10^{0}$ & 2.37 & $1.07 \times 10^{0}$ & 3.14 & $5.5 \times 10^{-1}$ & 2.69 & $2.0 \times 10^{-2}$ \\
\hline & calculated & & $1.26 \times 10^{0}$ & & $1.1 \times 10^{-0}$ & & $5.5 \times 10^{-1}$ & & $2.4 \times 10^{-2}$ \\
\hline \multirow[t]{2}{*}{ Rutile } & reference $^{4}$ & 2.31 & $1.2 \times 10^{0}$ & 3.17 & $8.4 \times 10^{-1}$ & 3.05 & 0 & 2.74 & 0 \\
\hline & calculated & & $1.1 \times 10^{0}$ & & $1.0 \times 10^{-1}$ & & $1.2 \times 10^{-4}$ & & $1.6 \times 10^{-4}$ \\
\hline \multirow[t]{2}{*}{ Quartz } & reference $^{5}$ & 1.60 & & 1.57 & & 1.55 & & 1.54 & \\
\hline & calculated & & $6.5 \times 10^{-3}$ & & $4.1 \times 10^{-3}$ & & $2.3 \times 10^{-3}$ & & $3.1 \times 10^{-3}$ \\
\hline \multirow[t]{2}{*}{ Limestone } & reference $^{5}$ & 1.68 & & 1.63 & & 1.61 & & 1.59 & \\
\hline & calculated & & $3.0 \times 10^{-3}$ & & $2.6 \times 10^{-3}$ & & $4.8 \times 10^{-4}$ & & $6.0 \times 10^{-4}$ \\
\hline
\end{tabular}

Sources of the complex refractive indices used in the calculations: (1) Egan and Hilgeman (1979); (2) Arakawa et al. (1997); (3) Querry (1985); (4) Sopra 2; (5) Ghosh (1999).

lengths of the tubes, there is a relatively large difference in the number size distribution measured directly at the exit of the buffer volume and the PA detection cell, especially for particles with a characteristic diameter above $1 \mu \mathrm{m}$. On the other hand, the corrected size distribution data also show that almost all particles below $1.2 \mu \mathrm{m}$ get into the PA detector. Furthermore, it is also important to note that neglecting the particle loss in the instrumentation modifies the calculated optical spectra quite drastically, as shown in Fig. 3. It affects not only their overall magnitude but also their shape as described in other works (Filep et al., 2013).

As it is expected, their MAC values are several orders of magnitude smaller than those of BC $\left(7.5 \pm 1.2 \mathrm{~m}^{2} \mathrm{~g}^{-1}\right.$ at $550 \mathrm{~nm}$ (Bond and Bergstrom, 2006)), $10 \pm 3.5 \mathrm{~m}^{2} \mathrm{~g}^{-1}$ at $405 \mathrm{~nm}$ (Cross et al., 2010)) and $\operatorname{BrC}\left(0.5-1.2 \mathrm{~m}^{2} \mathrm{~g}^{-1}\right.$ at $404 \mathrm{~nm}$ (Lack et al., 2012)). On the other hand, the PA signals were measured with a high signal to noise ratio even for the smallest MAC values; consequently all the presented data have high reliability.

However it is also worth noting that, for particulate matter with high scattering to absorption ratio, not only the signal to noise but also the signal to background ratio can also affect the reliability of the measured data. In our study, the ratio of the total photoacoustic signal to background signal was varied between 1.4 and 3.3 depending on the investigated components and the applied wavelengths. The signal to noise ratio was higher than 20 even in the less absorptive components such limestone and quartz; therefore, all the 


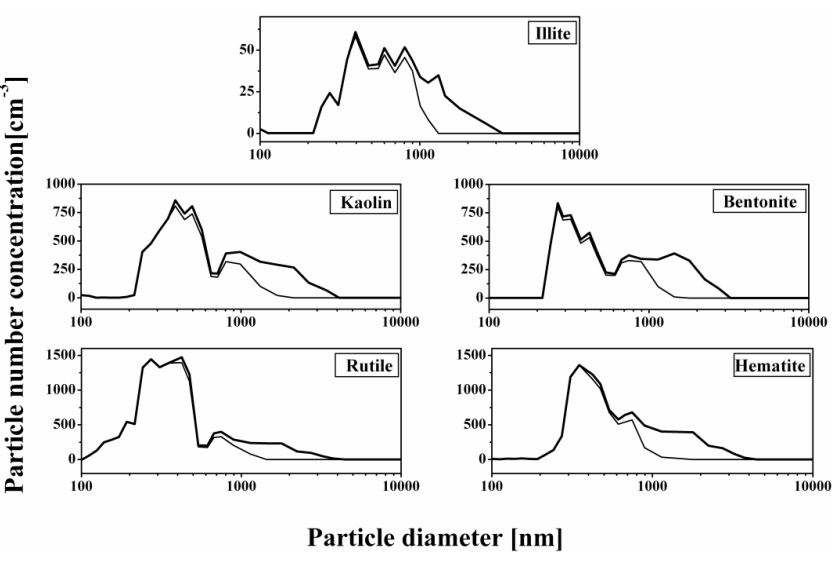

Figure 2. Number size distributions: in the buffer chamber measured by the SMPS and the OPC instruments (thick line) and in the PA cell calculated from the SMPS + OPC data by taking into account the calculated size transfer function of the PA system (thin line).

presented data have high reliability. The $\kappa$ values deduced from the presented measurements are compared to the values derived from the literature (Table 2). For some mineral dust components the differences between these values are within the combined uncertainty of the measurement and the calculation (e.g. in case of bentonite, hematite and rutile) while for other cases (such as for illite and kaolin) significant differences (above the uncertainty level) can be observed.

The uncertainties of the calculated parameters are affected by the fluctuations in the concentration of the generated aerosol, the noise of the PA measurements, the errors of the size distributions as well as the uncertainty of the literature values used in the retrieval procedure. In order to estimate the resulted uncertainty of the calculated parameters, a sensitivity study of the input parameters was performed. The uncertainty of the measured OAC values was determined directly by the uncertainty of the PA measurement including concentration instability. In case of MAC values the uncertainties of about \pm 20 and $\pm 5 \%$ were used for OAC and mass concentration measurements respectively, where the OAC uncertainty includes the concentration instability as well. The quadrature sum of these individual errors was found to be about $21 \%$. The uncertainty of the modelled OAC values is not determined here due to the lack of available information about the error of the literature $R i$ data. Error propagation of size distribution for the $R i$ retrieval was also investigated separately, and it was found that the uncertainty of the size distribution in between $\pm 15 \%$ translates directly into the computed $\chi$. Therefore, the resulting quadratic error of computed $\kappa$ was found to be about $25 \%$.

As far as the measured and the calculated OAC spectra shown in Fig. 3 are concerned, it must be first of all emphasized that the presented research does not intend to provide a quantitative comparison of the theoretical and experimental

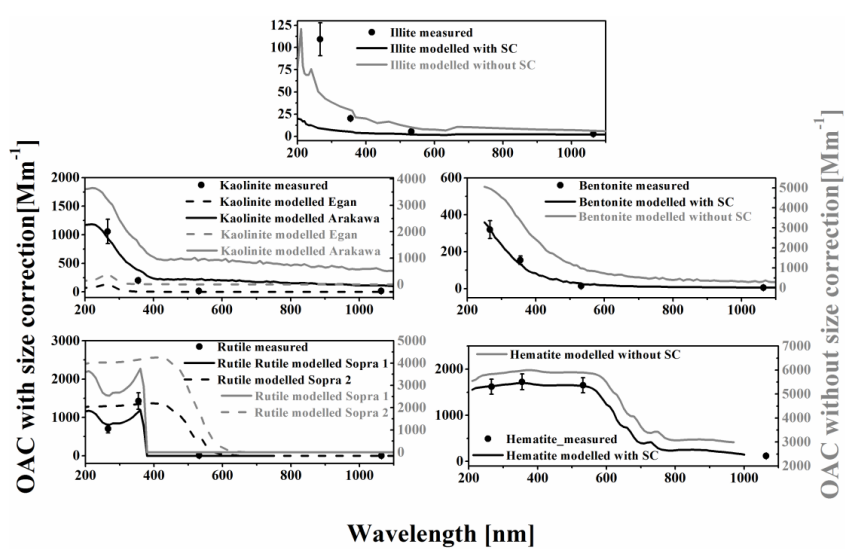

Figure 3. Aerosol optical absorption coefficients. The points represent OAC data calculated from PA signals with error bars representing the uncertainty of the OAC data (see text for details); the black and grey lines represent the Mie-theory-based calculations with or without size correction ( $\mathrm{SC}$ - taking into account the size transfer function of the PA system), respectively. The dashed grey lines represent the results of calculations by using alternative RI parameters (see text for details).

results or to validate the optical models or the $\kappa$ data used in the modelling because of the lack of detailed information on sample preparation and on measurement methods applied on bulk samples for the determination of $\kappa$. Rather, as the presented OAC values are based on a direct and aerosol-phase measurement of absorption using standardized measurement conditions and sample treatment, they can be used as reference values which reveal the deviation between the data presented earlier based on the differentiation of the applied methodology and the sample treatment. Moreover, they can contribute to the better understanding of the effect of bulkaerosol transition on the optical properties.

Despite all the uncertainties in the bulk measurements there is an overall order of magnitude agreement between the measured and the calculated optical absorption spectra shown in Fig. 3. In some cases (i.e. for bentonite and for hematite) the agreement is remarkably good whenever the size transfer function is taken into account. For other MD components there are various possible explanations for the deviations. In case of kaolinite the two presented calculations give rather different results and neither of them has a good agreement with the results of the PA measurement. The publication of Arakawa et al. (1997) criticized the measurement method of Egan and Hilgeman (1979) by stating that their photon detector had insufficient collection efficiency below $400 \mathrm{~nm}$. Indeed, the Arakawa et al. (1997) model shows much better agreement with the PA measurements in the short wavelength range than that of Egan and Hilgeman (1979). On the other hand, the calculation based on the data of Arakawa et al. (1997) overestimates the OAC compared to the PA results at longer wavelengths. As it was 

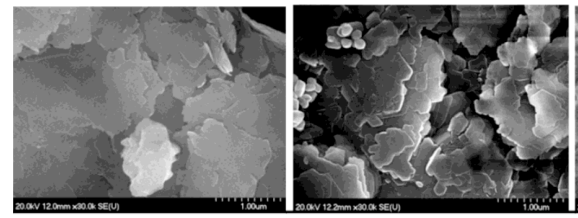

Illit

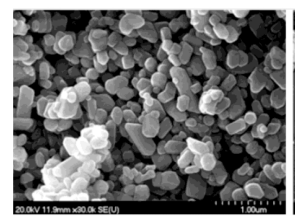

Rutile
Kaolin

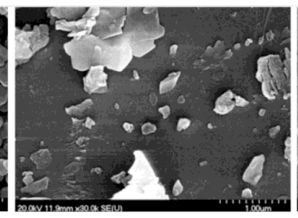

Quartz

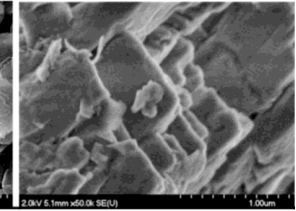

Bentonite

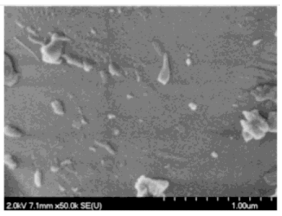

Limestone
Figure 4. SEM pictures of collected aerosol samples.

explained above, the low MAC values by the PA method still have high reliability; therefore, one can speculate that the overestimation by Egan and Hilgeman (1979) might be due to the fact that, in their transmission measurement made on a thin film, the wavelength of excitation in the long wavelength range became longer than the width of the film, and thus the light suffered multiple reflections, which resulted in the observed overestimation as described in detail elsewhere (McNeil and French, 2000; Arakawa et al., 1997). As far as illite is concerned, since it is measured with a similar method as kaolinite by Egan and Hilgeman (1979), the insufficient collection efficiency of their detector below $400 \mathrm{~nm}$ could again be a possible explanation for the discrepancies between the measurement and the calculation. However, the real differences between the measured and simulated values cannot be determined here due to the lack of revised data by Arakawa et al. (1997) in this case. Rutile provides a good example for the fact that even the same measurement method can result in strongly different spectra, most probably either due to differences in the sample preparation methods or due to differences in the samples themselves.

\section{Summary and conclusions}

The mass-specific optical absorption coefficient and the imaginary part of the complex refractive indices are determined for the most abundant MD components by using a recently developed multi-wavelength photoacoustic system. For modelling the OAC values by the Mie-theory-based simulation, we used the measured and particle-loss-corrected number size distribution, as well as tabulated $R i$ data based on bulk or thin film phase, an indirect measurement method taken from the published literature. Using the Mie-theorybased simulation and assuming spherical particles, we determined the refractive indices of the measured components and with the quoted limitations described in detail in Sect. 2.4.

We made a comparison between the measured and the simulated OAC data. We also experimentally demonstrated here that the reliability of both the measured and the simulated OAC values can be further increased by using particle-losscorrected measurement data.

In some cases we have found good agreement between the photoacoustically measured and the simulated OAC data, while in other cases we could give plausible explanations for the differences. Nevertheless, due to the limited availability of information on the applied measurement techniques, on sample preparation and the detailed description of the measurement conditions in case of bulk samples, the scientific goal of this work was not the quantitative comparison of the measured and the simulated OAC values. Rather, we wanted to measure aerosol-phase optical absorption using a direct and light-scattering immune technique. Due to being free from all analytical and methodological artefacts (which may occur when using competitive techniques), the values measured and presented here can be used for comparison with the optical absorption measurements conducted on bulk-phase samples. Furthermore, the data deduced from the presented experiments open up novel possibilities to experimentally investigate the effect of the bulk-aerosol transition when the bulk-phase data are used in aerosol forcing calculations.

Acknowledgements. Financial support by the Hungarian Scientific Research Foundation (OTKA, project no. K101905) is gratefully acknowledged. The European Union and the European Social Fund have provided financial support to the project under the project no. TÁMOP-4.2.2.A-11/1/KONV-2012-0047 and TÁMOP FUTURICT 4.2.2.C-11/1/KONV-2012-0013. The research of Noémi Utry was supported by the European Union and the State of Hungary, co-financed by the European Social Fund in the framework of TÁMOP 4.2.4. A/2-11-1-2012-0001 "National Excellence Program".

Edited by: F. Pope

\section{References}

Ajtai, T., Filep, Á., Kecskeméti, G., Hopp, B., Bozóki, Z., and Szabó, G.: Wavelength dependent mass-specific optical absorption coefficients of laser generated coal aerosols determined from multi-wavelength photoacoustic measurements, App. Phys. A, 103, 1165-1172, 2010.

Andreae, M. O.: The dark side of aerosols, Nature, 409, 671-672, 2001.

Andreae, M. O. and Gelencsér, A.: Black carbon or brown carbon? The nature of light-absorbing carbonaceous aerosols, Atmos. Chem. Phys., 6, 3131-3148, doi:10.5194/acp-6-3131-2006, 2006.

Arakawa, E. T., Tuminello, P. S., Khare, B. N., Millham, M. E., Authier, S., and Pierce, J.: Measurement of optical properties of small particles, ORNL/CP-95872, Oak Ridge National Lab, United States, 1997.

Bohor, B. F. and Hughes, R. E.: Scanning electron microscopy of clays and clay minerals, Clay Clay Min., 9, 49-54, 1971. 
Bond, T. C. and Bergstrom, R. W.: Light absorption by carbonaceous particles: An investigative review, Aerosol Sci. Technol., 40, 27-67, 2006.

Bozóki, Z., Pogány, A., and Szabó, G.: Photoacoustic instruments for practical applications: present, potentials, and future challenges, Appl. Spectr. Rev., 46, 1-37, 2011.

Cappa, C., Lack, D., Burkholder, J., and Ravishankara, A. R.: Bias in filter-based aerosol light absorption measurements due to organic aerosol loading: evidence from laboratory measurements, Aerosol Sci. Technol., 42, 1022-1032, 2008.

Chakrabarty, R. K., Moosmüller, H., Chen, L.-W. A., Lewis, K., Arnott, W. P., Mazzoleni, C., Dubey, M. K., Wold, C. E., Hao, W. M., and Kreidenweis, S. M.: Brown carbon in tar balls from smoldering biomass combustion, Atmos. Chem. Phys., 10, 63636370, doi:10.5194/acp-10-6363-2010, 2010.

Claquin, T., Schulz, M., Balkanski, Y., and Boucher, O.: Uncertainties in assessing radiative forcing by mineral dust, Tellus B, 50, 491-505, 1998.

Conant, W. C., Seinfeld, J. H., Wang, J., Carmichael, G. R., Tang, Y., Uno, I., Flatau, P. J., Markowicz, K. M., and Quinn, P. K.: A model for the radiative forcing during ACE-Asia derived from CIRPAS Twin Otter and R/V Ronald H. Brown data and comparison with observations, J. Geophys. Res., 108, 8661, doi:10.1029/2002JD003260, 2003.

Cross, E. S., Onasch, T. B., Ahern, A., Wrobel, W., Slowik, J. G., Olfert, J., Lack, D. A., Massoli, P., Cappa, C. D., Schwarz, J. P., Spackman, J. R., Fahey, D. W., Sedlacek, A., Trimborn, A., Jayne, J. T., Freedman, A., Williams, L. R., Ng, N. L., Mazzoleni, C., Dubey, M., Brem, B., Kok, G., Subramanian, R., Freitag, S., Clarke, A., Thornhill, D., Marr, L. C., Kolb, C. E., Worsnop, D. R., and Davidovits, P.: Soot particle studies - instrument intercomparison - project overview, Aerosol Sci. Technol., 44, 592611,2010

DeSouza-Machado, S. G., Strow, L. L., Hannon, S. E., and Motteler, H. E.: Infrared dust spectral signatures from AIRS, Geophys. Res. Lett., 33, L03801, doi:10.1029/2005GL024364, 2006.

Dong, Z., Qin, D., Kang, S., Ren, J., Chen, J., Cui, X., and Qin, X.: Physicochemical characteristics and sources of atmospheric dust deposition in snow packs on the glaciers of western Qilian Mountains, China, Tellus B, 66, 79-95, 2014.

Egan, W. G. and Hilgeman T. W.: Optical Properties of Inhomogeneous Materials: Applications to Geology, Astronomy, Chemistry, and Engineering, Academic, San Diego, Calif., 1979.

Filep, Á., Ajtai, T., Utry, N., Pintér, M. D., Nyilas, T., Takács, S., and Szabó, G.: Absorption Spectrum of Ambient Aerosol and Its Correlation with Size Distribution in Specific Atmospheric Conditions after a Red Mud Accident, Aerosol Air Qual. Res., 13, 49-59, 2013.

Ghosh, G.: Dispersion-equation coefficients for the refractive index and birefringence of calcite and quartz crystals, Opt. Commu., 163, 95-102, 1999.

Guyon, P., Boucher, O., Graham, B., Beck, J., Mayol-Bracero, O. L., Roberts, G. C., Maenhaut, W., Artaxo, P., and Andreae, M. O.: Refractive index of aerosol particles over the Amazon tropical forest during LBA-EUSTACH 1999, J. Aerosol. Sci., 34, 883907, 2003

Hoffer, A., Gelencsér, A., Guyon, P., Kiss, G., Schmid, O., Frank, G. P., Artaxo, P., and Andreae, M. O.: Optical properties of humic-like substances (HULIS) in biomass-burning aerosols, At- mos. Chem. Phys., 6, 3563-3570, doi:10.5194/acp-6-3563-2006, 2006.

Kalashnikova, O. V. and Sokolik, I. N.: Modeling the radiative properties of nonspherical soil-derived mineral aerosols, J. Quant. Spectr. Rad. Trans., 87, 137-166, 2004.

Lack, D. A., Langridge, J. M., Bahreini, R., Cappa, C. D., Middlebrook, A. M., and Schwarz, J. P.: Brown carbon and internal mixing in biomass burning particles, Proc. Natl. Acad. Sci., 109, 4802-14807, 2012.

McConnell, C. L., Formenti, P., Highwood, E. J., and Harrison, M. A. J.: Using aircraft measurements to determine the refractive index of Saharan dust during the DODO Experiments, Atmos. Chem. Phys., 10, 3081-3098, doi:10.5194/acp-10-30812010, 2010.

McNeil, L. E. and French, R. H.: Multiple scattering from rutile TiO2 particles, Actamaterialia, 48, 4571-4576, 2000.

Moffet, R. C. and Prather, K. A.: Extending ATOFMS measurements to include refractive index and density, Anal. Chem., 77, 6535-6541, 2005.

Moosmüller, H., Chakrabarty, R. K., and Arnott, W. P.: Aerosol light absorption and its measurement: A review, J. Quant. Spectr. Rad. Trans., 110, 844-878, 2009.

Moosmüller, H., Engelbrecht, J. P., Skiba, M., Frey, G., Chakrabarty R. K., and Arnott, W. P.: Single Scattering Albedo of Fine Mineral Dust Aerosols Controlled by Iron Concentration, J. Geophys. Res., 117, D11210, doi:10.1029/2011JD016909, 2012.

Müller, T., Schladitz, A., Massling, A., Kaaden, N., Kandler, K., and Wiedensohler, A.: Spectral absorption coefficients and imaginary parts of refractive indices of Saharan dust during SAMUM1. Tellus B, 61, 79-95, doi:10.3402/tellusb.v61i1.16816, 2011.

Nickovic, S., Vukovic, A., Vujadinovic, M., Djurdjevic, V., and Pejanovic, G.: Technical Note: High-resolution mineralogical database of dust-productive soils for atmospheric dust modeling, Atmos. Chem. Phys., 12, 845-855, doi:10.5194/acp-12-8452012, 2012.

Otto, S., de Reus, M., Trautmann, T., Thomas, A., Wendisch, M., and Borrmann, S.: Atmospheric radiative effects of an in situ measured Saharan dust plume and the role of large particles, Atmos. Chem. Phys., 7, 4887-4903, doi:10.5194/acp-7-4887-2007, 2007.

Patashnick, H., Meyer, M. and Rogers, B.: Tapered element oscillating microbalance technology Proc N American/Ninth U.S. Mine Vent Symp, June 2002, 625-631, 2002.

Querry, M. R.: Optical constants, MISSOURI UNIV-KANSAS CITY, 1985.

Rao, K. N.: Influence of deposition parameters on optical properties of TiO2 films, Opt. Eng., 41, 2357-2364, 2002.

Sokolik, I., Andronova, A., and Johnson, T., Complex refractive index of atmospheric dust aerosols, Atmos. Environ., 27A, 24952502, 1993.

Sokolik, I. N. and Toon, O. B.: Direct radiative forcing by anthropogenic airborne mineral aerosols, Nature, 381, 681-683, 1996.

Sokolik, I. N. and Toon, O.: Incorporation of mineralogical composition into models of the radiative properties of mineral aerosol from UV to IR wavelengths, J. Geophys. Res., 104, 9423-9444, 1999.

Tegen, I. and Fung, I.: Contribution to the atmospheric mineral aerosol load from land surface modification, J. Geophys. Res.Atmos., 100, 18707-18726, 1995. 
Tegen, I. and Lacis, A. A.: Modeling of particle size distribution and its influence on the radiative properties of mineral dust aerosol, J. Geophys. Res.-Atmos., 101, 19237-19244, 1996.

Utry, N., Ajtai, T., Pintér, M., Bozóki, Z., and Szabó, G.: Wavelength-Dependent Optical Absorption Properties of Artificial and Atmospheric Aerosol Measured by a Multi-Wavelength Photoacoustic Spectrometer, Int. J. Thermophys., 35, 22462258, 2014.

von der Weiden, S.-L., Drewnick, F., and Borrmann, S.: Particle Loss Calculator - a new software tool for the assessment of the performance of aerosol inlet systems, Atmos. Meas. Tech., 2, 479-494, doi:10.5194/amt-2-479-2009, 2009.
Wang, J., Flagan, R. C., Seinfeld, J. H., Jonsson, H. H., Collins, D. R., Russell, P. B., Schmid, B., Redemann, J., Livingston, J. M., Gao, S., Hegg, D. A., Welton, E. J., and Bates, D.: Clearcolumn radiative closure during ACE-Asia: Comparison of multiwavelength extinction derived from particle size and composition with results from Sunphotometry, J. Geophys. Res., 107, 4688, doi:10.1029/2002JD002465, 2002. 